Article

\title{
A Facile Synthesis of Functionalized Dispirooxindole Derivatives via a Three-Component 1,3-Dipolar Cycloaddition Reaction
}

\author{
Jun He ${ }^{1, \dagger}$, Guang Ouyang ${ }^{2, \dagger}$, Zhixiang Yuan ${ }^{3}$, Rongsheng Tong ${ }^{2}$, Jianyou Shi ${ }^{2, *}$ and
} Liang Ouyang ${ }^{1, *}$

1 State Key Laboratory of Biotherapy, West China Hospital, Sichuan University, Chengdu 610041, China

2 Sichuan Academy of Medical Sciences, Sichuan Provincial People's Hospital, Chengdu 610072, China

3 Institute of Pharmacy, Sichuan Academy of Chinese Medicine Sciences, Chengdu 610041, China

$\dagger$ These authors contributed equally to this work.

* Authors to whom correspondence should be addressed; E-Mails: shijianyoude@126.com (J.S.); ouyangliang@scu.edu.cn (L.O.); Tel.: +86-028-8739-3234 (J.S.); Fax: +86-028-8550-3817 (L.O.).

Received: 3 April 2013; in revised form: 26 April 2013 / Accepted: 27 April 2013 /

Published: 3 May 2013

\begin{abstract}
An efficient synthesis of novel dispirooxindoles has been achieved through three-component 1,3-dipolar cycloaddition of azomethine ylides generated in situ by the decarboxylative condensation of isatin and an $\alpha$-amino acid with the dipolarophile 5-benzylideneimidazolidine-2,4-dione. The improved procedure features mild reaction conditions, high yields, high diastereoselectivities, a one-pot procedure and operational simplicity.
\end{abstract}

Keywords: multicomponent reactions; 1,3-dipolar cycloaddition; azomethine ylide; dispirooxindole

\section{Introduction}

Nowadays, conventional one-pot, multicomponent reactions (MCRs) are considered to be one of the most efficient strategies in organic and medical chemistry for synthesizing structurally diverse compounds and biologically active natural products, usually in a stereoselective-manner [1]. The 
highly effective one-pot procedure of MCRs exhibits many advantages, including atom economy, facile synthesis, convergence, productivity and easy execution [2]. MCRs were a common method for building molecular diversities with complex scaffolds and had broad applications in combinatorial chemistry, which allowed a rapid access to identify a promising lead molecule in drug candidate discovery [3-5].

The 1,3-dipolar cycloaddition of azomethine ylides with olefinic and acetylenic dipolarophiles is one of the most useful MCRs for providing an useful approach to building nitrogen-containing fivemembered ring heterocycles, such as pyrroline, pyrrolidine, pyrrolizidine or spirooxindole derivatives [6-9], which served as useful molecular scaffolds for the exploration and exploitation of pharmacophore space via diversity-oriented synthesis [10-13]. Among of them, dispirooxindole ring systems possess more interesting structural properties and have been reported to exhibit strong bioactivity profiles including antimicrobial [14], antitumoral [15], anti-inflammatory [16], anti-HIV [17] and potent non-peptide inhibition of the p53-MDM2 interaction [18].

Hydantoin derivatives are widely used in malignant hyperthermia, neuroleptic malignant syndrome, spasticity, and anticonvulsants [19-22], especially the spirohydantoins, which are considered to be a novel aldose reduetase inhibitor to treat for diabetes [23]. Significant efforts have been focused on developing a general synthetic route to access those compounds. However, to the best of our knowledge, only a few methods were reported to synthesize the spiropyrrolidine bisoxindoles [24,25], and a general method to prepare dispirooxindole hydantoin derivatives is still lacking. Thus, in order to extend our interest in cycloaddition reactions of novel spiro compounds and nitrogen heterocycles with biological activities [26-28], we report herein the efficient synthesis in excellent yields of a series of novel dispirooxindole derivatives by the three-component 1,3-dipolar cycloaddition reaction of nonstabilized azomethine ylides generated in situ by the decarboxylative condensation of isatin and primary $\alpha$-amino acid with the Knoevenagel adduct derivatives (preformed by reaction of hydantoin with substituted benzaldehydes).

\section{Results and Discussion}

In our initial endeavor, the Knoevenagel adducts 3 were synthesized via a method involving condensation of commercially available hydantoin and substituted benzaldehydes in water using ethanolamine as catalyst. After work-up, the crude reaction mixtures were purified by recrystallization in ethanol/water (40:60) to afford 63-99\% total yields of the target products (Scheme 1). Melting point, NMR and mass spectrometry data were consistent with those reported in the literature [29].

Scheme 1. The synthetic route to compounds 3 .

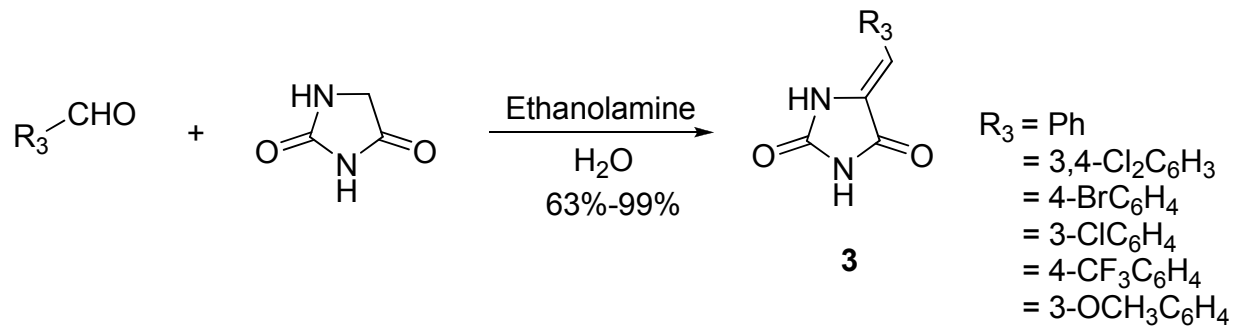


From the mechanistic perspective, the azomethine ylides, a powerful class of reagents, have featured in a number of 1,3-dipolar cycloaddition reactions. In combination with the experiences from our previous work, we envisaged that an azomethine ylide could be generated in situ from isatin (1a) and L-proline (2a), and then trapped with Knoevenagel adduct 5-benzylideneimidazolidine-2,4-dione (3a) acting as dipolarophile, to afford spiropyrrolizidine oxindole 4a. Hence, the 1,3-dipolar cycloaddition reaction would be facilitated in one-pot with two steps. Although the azomethine ylides, generated from the reaction of isatin and L-proline, have two nucleophilic carbons potentially resulting in two regioisomers. However, high regioselectivity was observed in the formation of the product (see below). It may resulted from the more stable transition state (Inta) leading to the observed products (4a). Meanwhile, the other possible one (Intb) would be less stable because of steric interactions between the aryl ring of Knoevenagel adducts and isatin backbone (Scheme 2).

Scheme 2. Possible reaction mechanism for the synthesis of dispirooxindole hydantoin derivatives.<smiles>O=C1Nc2ccccc2C1=O</smiles>

$1 a$<smiles>O=C1NC(=O)/C(=C/c2ccccc2)NC1=O</smiles><smiles>O=C(O)[C]1CCCN1</smiles>

$2 \mathbf{a}$<smiles>CC(C)(C)C1(C(=O)O)CCCN1C1C(=O)Nc2ccccc21</smiles>

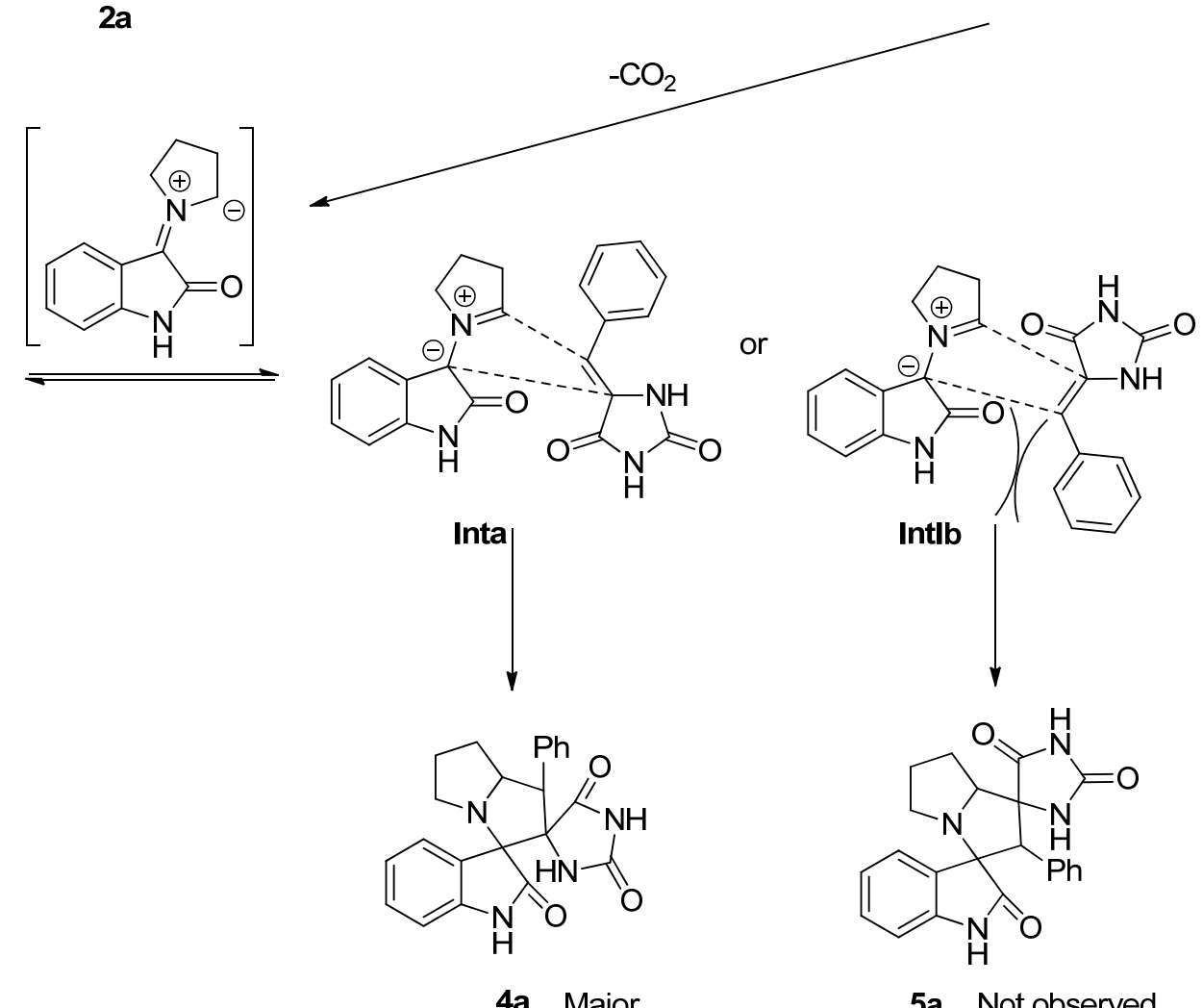

5a Not observed 
In an effort to identify the reaction parameters of this one-pot process, the three-component reaction of isatin, L-proline and 5-benzylideneimidazolidine-2,4-dione was carried out as model reaction. Firstly, various solvents were examined under $80{ }^{\circ} \mathrm{C}$, and the results were summarized in Table 1. Acetonitrile and ether solvents such as dioxane and THF gave moderate yields (entries 1-3), and with toluene as solvent, a poor yield resulted (entry 4). To our delight, the alcohol solvent methanol gave promising yields, and ethanol afforded even better results (entries 5-6). Ethanol may facilitate the production of the azomethine ylide by accelerating the formation of an iminium species between isatin and L-proline. Then the reaction temperature, mixed solvent and time were further investigated, the desired product was finally obtained as a single regioisomer in almost quantitative yield (95\%) after 10 hours at $50{ }^{\circ} \mathrm{C}$ (entries 7-9). Consequently, we chose these conditions for the rest of our studies.

Table 1. Optimization of reaction conditions ${ }^{\text {a }}$.

\begin{tabular}{llll}
\hline Entry & Solvent & Temp $\left({ }^{\circ} \mathbf{C}\right)$ & Yield $^{\mathbf{b}} \mathbf{( \% )}$ \\
\hline 1 & 1,4-dioxane & 80 & 33 \\
2 & THF & 80 & 42 \\
3 & $\mathrm{CH}_{3} \mathrm{CN}$ & 80 & 31 \\
4 & Toluene & 80 & 17 \\
5 & Methanol & reflux & 57 \\
6 & Ethanol & 80 & 68 \\
7 & Ethanol $/ \mathrm{H}_{2} \mathrm{O}$ & 100 & 55 \\
8 & Ethanol & 50 & 74 \\
$9^{\mathrm{c}}$ & Ethanol & 50 & 95 \\
\hline
\end{tabular}

${ }^{a}$ Unless indicated otherwise, the reaction was performed with $\mathbf{3 a}(0.5 \mathrm{mmol}), \mathbf{1 a}$ isatin $(0.5 \mathrm{mmol})$, and L-proline $(0.5 \mathrm{mmol})$ in different solvents $(10.0 \mathrm{~mL})$ and temperatures for $5 \mathrm{~h} .{ }^{\mathrm{b}}$ Isolated yield based on isatin. ${ }^{\mathrm{c}} 10 \mathrm{~h}$.

With the optimized reaction conditions in hand, various structurally diverse 5-benzylideneimidazolidine-2,4-diones $\mathbf{3}$ were investigated. Gratifyingly, the corresponding spiro-pyrrolidine products $\mathbf{4 a}-\mathbf{4 m}$ were obtained as single diastereoisomers in high yields. As shown in Table 2, different substitutents on the aryl ring, such as bromo, chloro, $\mathrm{CF}_{3}$ and $\mathrm{OCH}_{3}$ groups at the meta or para positons all gave corresponding products $\mathbf{4 e}, \mathbf{4 i}, \mathbf{4 l}$ and $\mathbf{4 m}$ in high yields ranging from $85 \%$ to 90\% (entries 5, 9, 12 and 13). Furthermore, different substituents in the isatin such as 5-F, - $\mathrm{Cl}$ or $-\mathrm{Br}$ also reacted smoothly to generate the desired products in high yields (entries 3, 6, 7 and 10). More interestingly, the unprotected isatin gave even better results (entry 1 vs. 2), which opens a door to further functionalize the products in future medicinal chemistry studies.

We also studied the cycloaddition reaction of the different amino acid thioproline $\mathbf{2} \mathbf{b}$, wbereby the reaction between isatin $\mathbf{1}, \mathbf{2 b}$ and $\mathbf{3}$ happened smoothly to afford the desired spirothiopyrrolidine $\mathbf{4 n}$ as a single diastereoisomer in $90 \%$ yield (Scheme 3). 
Table 2. Scope of the reaction.

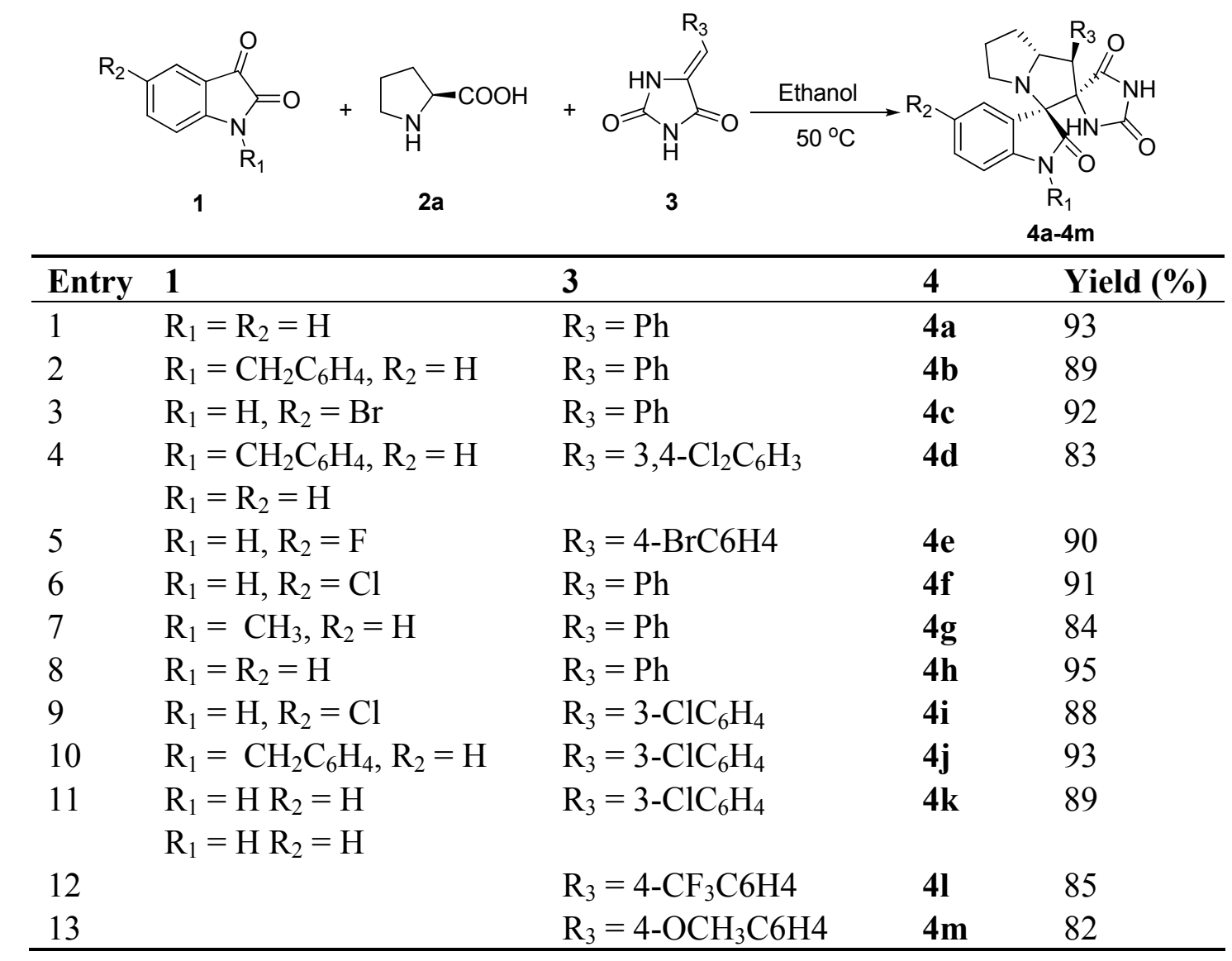

Scheme 3. MCRs of thioproline $\mathbf{2 b}$.

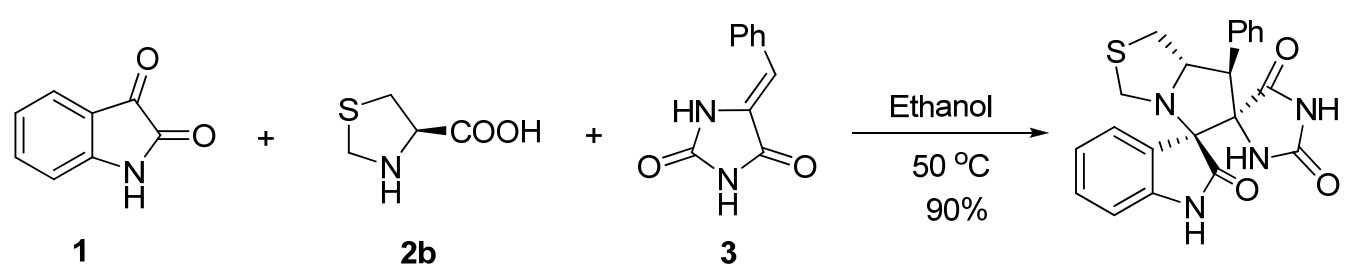

$4 n$

To further confirm the structure, diastereoselectivity and regioselectivity, detailed NMR spectral and X-ray analyses were carried out. The structures proposed for all products were in agreement with their NMR spectra, as discussed for compound $\mathbf{4 b}$ as an example In the ${ }^{1} \mathrm{H}-\mathrm{NMR}$ spectrum of $\mathbf{4 b}$, the pyrrolidine ring proton of C-5 exhibited a multiplet $(\mathrm{m})$ peak at $\delta 4.48(\mathrm{~m}, 1 \mathrm{H})$. The $\mathrm{C}-4$ proton which was attached to the aryl group appeared as a doublet at $\delta 3.77(\mathrm{~d}, J=10.3 \mathrm{~Hz}, 1 \mathrm{H})$. The aromatic protons were distributed in the $\delta 7.41-6.24$ region. The NH proton appeared as a singlet at $\delta 8.37$ and $\delta$ 10.37. Based on the calculation of the coupling constant ( $J$-based configuration analysis, $\mathrm{J}>10 \mathrm{~Hz}$ ), the relative configuration of this structure should be as same as compound $\mathbf{4 b}$ shown in Figure 1 and the configuration was further confirmed by the X-ray study of a single crystal of compound $\mathbf{4 b}$ (Figure $1 \mathrm{~b}$ ). The results revealed that the pyrrolidine ring adopted an envelope form with the spiro carbon being out of plane. The ${ }^{13} \mathrm{C}-\mathrm{NMR}$ of compound $\mathbf{4 b}$ supported the proposed structure as well. The pyrrolidine ring carbons resonated in the $\delta 67.67 \mathrm{ppm}$ region. The carbonyl carbon resonated at $\delta 154.93 \mathrm{ppm}$, respectively. 
Figure 1. (a) Selected ${ }^{1} \mathrm{H}$ - and ${ }^{13} \mathrm{C}-\mathrm{NMR}$ chemical shifts of $4 \mathbf{b}$. (b) Single crystal $\mathrm{X}$-ray diffraction study of compound $\mathbf{4 b}$.

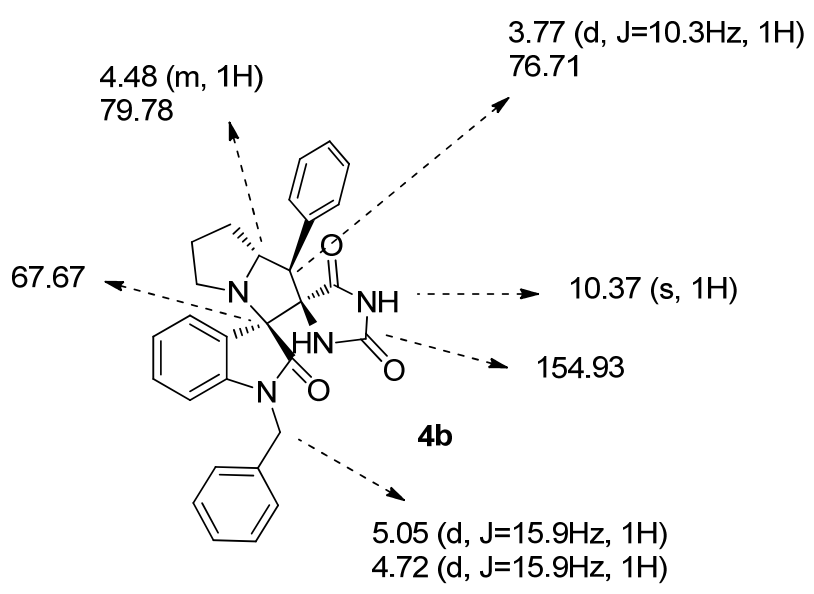

(a)

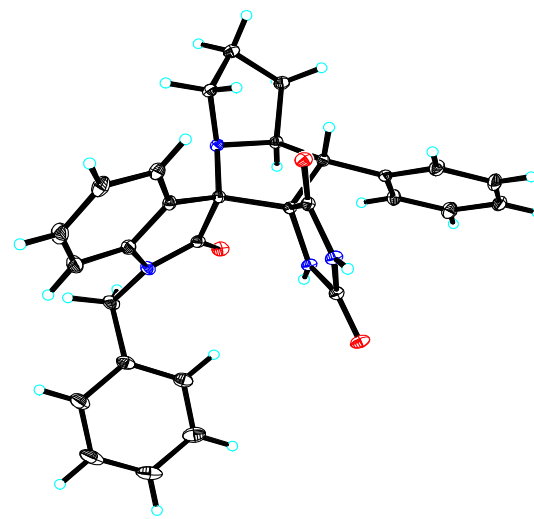

(b)

The regioselectivity in formation of $\mathbf{4}$ can be explained by considering the secondary orbital interaction (SOI) mechanism proposed in Scheme 4 [30]. The reaction proceeds through the generation of azomethine ylide via the condensation of isatin with L-proline and decarboxylation. The dipolarophile 3 regioselectively reacts with azomethine ylides in ethanol to give the desired products compounds 4 . The X-ray structure of the product $\mathbf{4 b}$ reflects that the cycloaddition proceeds via an exo'-transition state (Scheme 4, path B). This can be explained by the fact that the corresponding endo'-transition state (A) would require more free energy of activation than the exo'-transition state (B) leading to $\mathbf{4} \mathbf{a}$ as the former would result in electrostatic repulsion between the cis carbonyls increasing the free energy of activation. Accordingly, the observed regioisomer 4 via path B is more favorable because of the SOI which is not possible in path A [31].

Scheme 4. Plausible mechanism for the formation of compound 4.

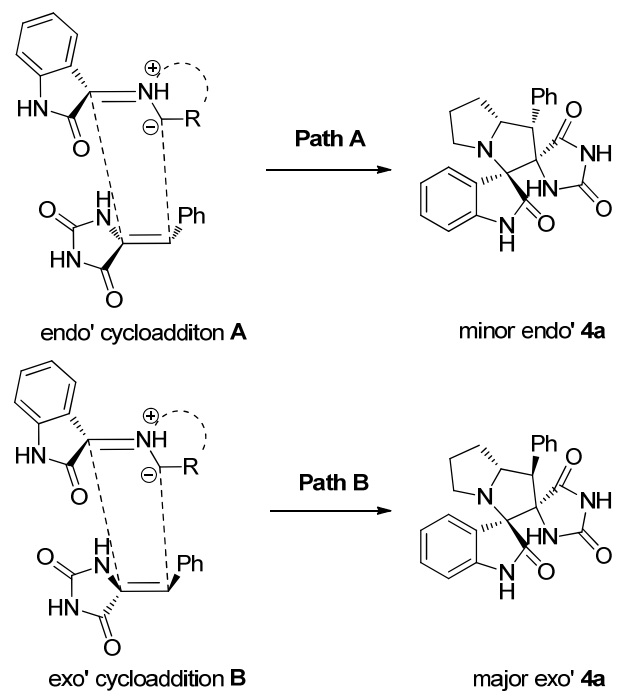




\section{Experimental}

\subsection{General}

All reagents were purchased from commercial sources and used without further purification. Melting points are corrected. ${ }^{1} \mathrm{H}-\mathrm{NMR}$ spectra were determined on a Bruker Avance III $400 \mathrm{MHz}$ spectrometer in DMSO- $\mathrm{d}^{6}$ solution. $\mathrm{J}$ values are in Hz. Chemical shifts are expressed in ppm downfield from internal standard TMS. HRMS data were obtained using Bruker micrOTOF-Q instrument or TOF-MS instrument. The starting compounds $\mathbf{3}$ were prepared according to the previously reported procedures.

\subsection{General Procedure for the Synthesis of Dispirooxindoles 4}

A dry $50 \mathrm{~mL}$ flask was charged with istain derivatives $\mathbf{1}(0.5 \mathrm{mmol})$, L-proline, L-thioproline $\mathbf{2 a}$ or 2b $(0.5 \mathrm{mmol})$, and imidazolidin-2-one derivatives $3(0.5 \mathrm{mmol})$, and ethanol $(10 \mathrm{~mL})$. The mixture was stirred at $50{ }^{\circ} \mathrm{C}$ for $10 \mathrm{~h}$. After completion of the reaction (monitored by TLC), the solvent was cooled, then was filtrated and washed by $10 \mathrm{~mL}$ of ethanol twice to give solid. The solid was dried at $80{ }^{\circ} \mathrm{C}$ for $4 \mathrm{~h}$ under vacuum to give compounds 4 . The structures of the products were identified by ${ }^{1} \mathrm{H}-\mathrm{NMR},{ }^{13} \mathrm{C}\{1 \mathrm{H}\}-\mathrm{NMR}$ and HRMS spectra. The structure and regiochemistry of the products were assigned on the basis of their spectroscopic analysis.

1-Phenylhexahydro-1H-pyrrolizine-2-spiro-5'-imidazolidine-2',4'-dione-3-spiro-3"-indoline-2"-one (4a). White solid; m.p. 196-198 ${ }^{\circ} \mathrm{C}$; ${ }^{1} \mathrm{H}-\mathrm{NMR}$ (DMSO-d $\left.)_{6}\right): \delta$ (ppm) 1.56-1.69 (m, 1H, $\mathrm{CH}_{2}$ ), 1.73-2.06 (m, 3H, CH $)$, 2.81-2.95 (m, 1H, $\left.\mathrm{CH}_{2}\right), 3.70$ (d, $\left.J=10.3 \mathrm{~Hz}, 1 \mathrm{H}, \mathrm{CH}\right), 4.31-4.52(\mathrm{~m}, 1 \mathrm{H}$, $\mathrm{CH}), 6.80(\mathrm{~d}, J=7.6 \mathrm{~Hz}, 1 \mathrm{H}, \mathrm{ArH}), 6.98-7.07(\mathrm{~m}, 1 \mathrm{H}, \mathrm{ArH}), 7.19-7.34(\mathrm{~m}, 4 \mathrm{H}, \mathrm{ArH}), 7.36(\mathrm{~d}$, $J=7.1 \mathrm{~Hz}, 2 \mathrm{H}, \mathrm{ArH}), 7.50$ (d, $J=7.5 \mathrm{~Hz}, 1 \mathrm{H}, \mathrm{ArH}), 8.10$ (s, 1H, NH), 10.33 (s, 1H, NH), 10.42 (s, $1 \mathrm{H}, \mathrm{NH}) ;{ }^{13} \mathrm{C}\{1 \mathrm{H}\}-\mathrm{NMR}\left(\mathrm{DMSO}-\mathrm{d}_{6}\right): \delta 27.19,29.56,46.43,55.99,56.79,67.50,76.79,79.47$, $109.78,120.98,124.21,127.36,128.20,128.32$, 129.31, 129.66, 134.80, 154.93, 172.39, 176.38; HRMS: calcd. for $\mathrm{C}_{22} \mathrm{H}_{20} \mathrm{~N}_{4} \mathrm{O}_{3}{ }^{+}[\mathrm{M}+\mathrm{H}]^{+}: 389.1613$, found: 389.1614 .

1-Phenylhexahydro-1H-pyrrolizine-2-spiro-5'-imidazolidine-2',4'-dione-3-spiro-3"-N-benzylindoline2"-one (4b). White solid; m.p. 187-189 ${ }^{\circ} \mathrm{C}$; ${ }^{1} \mathrm{H}-\mathrm{NMR}$ (DMSO-d $)_{6}$ : $\delta(\mathrm{ppm}) 1.51-1.73\left(\mathrm{~m}, 1 \mathrm{H}, \mathrm{CH}_{2}\right.$ ), 1.75-2.10 (m, 3H, $\left.\mathrm{CH}_{2}\right), 2.81-2.92\left(\mathrm{~m}, 1 \mathrm{H}, \mathrm{CH}_{2}\right) .3 .77(\mathrm{~d}, J=10.3 \mathrm{~Hz}, 1 \mathrm{H}, \mathrm{CH}), 4.33-4.41$ (brs, $1 \mathrm{H}$, $\left.\mathrm{CH}_{2}\right), 4.43-4.53(\mathrm{~m}, 1 \mathrm{H}, \mathrm{CH}), 4.72\left(\mathrm{~d}, J=15.9 \mathrm{~Hz}, 1 \mathrm{H}, \mathrm{CH}_{2}\right), 5.05\left(\mathrm{~d}, J=15.9 \mathrm{~Hz}, 1 \mathrm{H}, \mathrm{CH}_{2}\right), 6.82(\mathrm{~d}$, $J=7.7 \mathrm{~Hz}, 1 \mathrm{H}, \mathrm{ArH}), 7.01-7.14(\mathrm{~m}, 1 \mathrm{H}, \mathrm{ArH}), 7.18-7.35$ (m, 9H, ArH), 7.36-7.46 (d, $J=6.9 \mathrm{~Hz}, 2 \mathrm{H}$, $\operatorname{ArH}), 7.59$ (d, $J=7.2 \mathrm{~Hz}, 1 \mathrm{H}, \mathrm{ArH}), 8.37$ (s, 1H, NH), 10.37 (s, 1H, NH); ${ }^{13} \mathrm{C}\{1 \mathrm{H}\}-\mathrm{NMR}\left(\mathrm{DMSO}-\mathrm{d}_{6}\right)$ : $\delta$ 27.47, 29.67, 42.75, 46.49, 56.06, 67.67, 76.71, 79.78, 109.35, 121.77, 123.69, 126.96, 127.35, 127.51, 128.28, 128.31, 129.4, 129.78, 134.67, 136.12, 142.46, 154.93, 172.15, 174.95; HRMS: calcd. for $\mathrm{C}_{29} \mathrm{H}_{26} \mathrm{~N}_{4} \mathrm{O}_{3}{ }^{+}[\mathrm{M}+\mathrm{H}]^{+}:$479.2080, found: 479.2083 .

1-Phenylhexahydro-1H-pyrrolizine-2-spiro-5'-imidazolidine-2',4'-dione-3-spiro-3"-5-bromoindoline2"-one (4c). White solid; m.p. 175-177 ${ }^{\circ} \mathrm{C} ;{ }^{1} \mathrm{H}-\mathrm{NMR}$ (DMSO-d 6 ): $\delta(\mathrm{ppm})$ 1.57-1.70 (m, 1H, $\mathrm{CH}_{2}$ ), 1.75-2.08 (m, 3H, $\left.\mathrm{CH}_{2}\right), 2.73-2.90\left(\mathrm{~m}, 1 \mathrm{H}, \mathrm{CH}_{2}\right), 3.66(\mathrm{~d}, J=10.5 \mathrm{~Hz}, 1 \mathrm{H}, \mathrm{CH}), 4.34-4.46(\mathrm{~m}, 1 \mathrm{H}$, $\mathrm{CH}), 6.75(\mathrm{~d}, J=8.3 \mathrm{~Hz}, 1 \mathrm{H}, \mathrm{ArH}), 7.23-7.34(\mathrm{~m}, 3 \mathrm{H}, \mathrm{ArH}), 7.37$ (d, $J=7.0 \mathrm{~Hz}, 2 \mathrm{H}, \mathrm{ArH}), 7.45$ (dd, $J=8.3,1.5 \mathrm{~Hz}, 1 \mathrm{H}, \mathrm{ArH}), 7.60$ (s, 1H, ArH), 8.29 (s, 1H, NH), 10.44 (s, 1H, NH), 10.59 (s, 1H, NH); 
${ }^{13} \mathrm{C}\{1 \mathrm{H}\}-\mathrm{NMR}\left(\mathrm{DMSO}-\mathrm{d}_{6}\right.$ ): $\delta 27.35,29.52,46.45,56.74,67.50,76.87,79.65,111.79,112.98,126.70$, 127.48, 128.24, 129.71, 130.67, 132.28, 134.55, 141.52, 154.85, 172.34, 175.93; HRMS: calcd. for $\mathrm{C}_{22} \mathrm{H}_{19} \mathrm{BrN}_{4} \mathrm{O}_{3}{ }^{+}[\mathrm{M}+\mathrm{H}]^{+}: 467.0728$, found: 467.0719.

1-(3,4-Dichloro)phenylhexahydro-1H-pyrrolizine-2-spiro-5'-imidazolidine-2',4'-dione-3-spiro-3"- $N$ benzylindoline-2"-one (4d). White solid; m.p. 160-162 ${ }^{\circ} \mathrm{C}$; ${ }^{1} \mathrm{H}-\mathrm{NMR}$ (DMSO- $\mathrm{d}_{6}$ ): $\delta$ (ppm) 1.53-1.67 $\left(\mathrm{m}, 1 \mathrm{H}, \mathrm{CH}_{2}\right), 1.68-1.80\left(\mathrm{~m}, 1 \mathrm{H}, \mathrm{CH}_{2}\right), 1.95-2.11\left(\mathrm{~m}, 2 \mathrm{H}, \mathrm{CH}_{2}\right), 2.62\left(\mathrm{t}, J=7.2 \mathrm{~Hz}, 1 \mathrm{H}, \mathrm{CH}_{2}\right)$, $3.45-3.55\left(\mathrm{~m}, 1 \mathrm{H}, \mathrm{CH}_{2}\right), 4.25(\mathrm{~d}, J=8.0 \mathrm{~Hz}, 1 \mathrm{H}, \mathrm{CH}), 4.66-4.79\left(\mathrm{~m}, 2 \mathrm{H}, 1 / 2 \mathrm{CH}_{2}, \mathrm{CH}\right), 5.05(\mathrm{~d}$, $\left.J=15.7 \mathrm{~Hz}, 1 \mathrm{H}, \mathrm{CH}_{2}\right), 6.84(\mathrm{~d}, J=7.8 \mathrm{~Hz}, 1 \mathrm{H}, \mathrm{ArH}), 7.01-7.10(\mathrm{~m}, 1 \mathrm{H}, \mathrm{ArH}), 7.21-7.31(\mathrm{~m}, 4 \mathrm{H}$, $\operatorname{ArH}), 7.34(\mathrm{~d}, J=7.1 \mathrm{~Hz}, 2 \mathrm{H}, \mathrm{ArH}), 7.53(\mathrm{dd}, J=8.4,2.3 \mathrm{~Hz}, 2 \mathrm{H}, \mathrm{ArH}), 7.63$ (d, $J=2.2 \mathrm{~Hz}, 1 \mathrm{H}$, ArH), 7.87 (s, 1H, ArH), $7.98(\mathrm{~d}, J=8.6 \mathrm{~Hz}, 1 \mathrm{H}, \mathrm{ArH}), 10.68(\mathrm{~s}, 1 \mathrm{H}, \mathrm{NH}) ;{ }^{13} \mathrm{C}\{1 \mathrm{H}\}-\mathrm{NMR}$ (DMSO$\left.\mathrm{d}_{6}\right): \delta 25.05,29.22,42.62,47.58,53.38,68.27,76.75,78.47,109.57,122.27,123.31,127.42,127.84$, $128.47,128.64,130.04,132.49,132.63,133.20,135.48,135.83,143.65,155.35,174.80,175.40$; HRMS: calcd. for $\mathrm{C}_{29} \mathrm{H}_{24} \mathrm{Cl}_{2} \mathrm{~N}_{4} \mathrm{O}_{3}{ }^{+}[\mathrm{M}+\mathrm{H}]^{+}: 547.1300$, found: 547.1304 .

1-(4-Bromo)phenylhexahydro-1H-pyrrolizine-2-spiro-5'-imidazolidine-2',4'-dione-3-spiro-3"'-indoline2"-one (4e). White solid; m.p. 222-224 ${ }^{\circ} \mathrm{C} ;{ }^{1} \mathrm{H}-\mathrm{NMR}$ (DMSO-d $\left.)_{6}\right): \delta(\mathrm{ppm}) 1.59-1.70\left(\mathrm{~m}, 1 \mathrm{H}, \mathrm{CH}_{2}\right)$, 1.72-2.05 (m, 3H, $\left.\mathrm{CH}_{2}\right), 2.83-2.96\left(\mathrm{~m}, 1 \mathrm{H}, \mathrm{CH}_{2}\right), 3.70(\mathrm{~d}, J=10.3 \mathrm{~Hz}, 1 \mathrm{H}, \mathrm{CH}), 4.32-4.40(\mathrm{~m}, 1 \mathrm{H}$, $\mathrm{CH}), 6.80(\mathrm{~d}, J=7.6 \mathrm{~Hz}, 1 \mathrm{H}, \mathrm{ArH}), 6.97-7.06(\mathrm{~m}, 1 \mathrm{H}, \mathrm{ArH}), 7.19-7.28(\mathrm{~m}, 1 \mathrm{H}, \mathrm{ArH}), 7.33(\mathrm{~d}, J=8.5$ $\mathrm{Hz}, 2 \mathrm{H}, \mathrm{ArH}), 7.43-7.59$ (m, 3H, ArH), 8.21 (s, 1H, NH), 10.41 (s, 1H, NH), 10.45 (s, 1H, NH); ${ }^{13} \mathrm{C}\{1 \mathrm{H}\}$-NMR (DMSO-d 6 ): $\delta 27.13,29.46,46.52,56.26,67.60,76.78,79.40,109.86,120.86,121.06$, 124.13, 128.37, 129.44, 131.16, 131.92, 134.34, 142.22, 155.01, 172.46, 176.34; HRMS: calcd. for $\mathrm{C}_{22} \mathrm{H}_{19} \mathrm{BrN}_{4} \mathrm{O}_{3}^{+}[\mathrm{M}+\mathrm{H}]^{+}: 467.0718$, found: 467.0719 .

1-Phenylhexahydro-1H-pyrrolizine-2-spiro-5'-imidazolidine-2',4'-dione-3-spiro-3"-5-fluoroindoline2"-one (4f). White solid; m.p. 196-197 ${ }^{\circ} \mathrm{C} ;{ }^{1} \mathrm{H}-\mathrm{NMR}$ (DMSO- $\left.\mathrm{d}_{6}\right)$ : $\delta$ (ppm) 1.59-1.74 (m, $1 \mathrm{H}, \mathrm{CH}_{2}$ ), 1.76-1.94 (m, 2H, $\left.\mathrm{CH}_{2}\right), 1.95-2.071\left(\mathrm{~m}, 1 \mathrm{H}, \mathrm{CH}_{2}\right), 2.72-2.83\left(\mathrm{~m}, 1 \mathrm{H}, \mathrm{CH}_{2}\right), 3.71(\mathrm{~d}$, $J=10.4 \mathrm{~Hz}, 1 \mathrm{H}, \mathrm{CH}), 4.33-4.43(\mathrm{~m}, 1 \mathrm{H}, \mathrm{CH}), 6.79$ (dd, $J=8.5,4.6 \mathrm{~Hz}, 1 \mathrm{H}, \mathrm{ArH}), 7.06-7.14(\mathrm{~m}, 1 \mathrm{H}$, ArH), 7.22-7.33 (m, 3H, ArH), 7.34-7.43 (m, 3H, ArH), 8.35 (s, 1H, NH), 10.37 (s, 1H, NH), 10.43 $(\mathrm{s}, 1 \mathrm{H}, \mathrm{NH}) ;{ }^{13} \mathrm{C}\{1 \mathrm{H}\}-\mathrm{NMR}\left(\mathrm{DMSO}-\mathrm{d}_{6}\right): \delta 27.74,29.63,46.26,56.35,67.52,77.11,79.72,110.45$, $110.53,115.71,115.95,116.21,126.02,126.10,127.48,128.19,129.73,134.60,138.25,154.89$, 156.72, 158.62, 171.89, 176.34; HRMS: calcd. for $\mathrm{C}_{22} \mathrm{H}_{19} \mathrm{FN}_{4} \mathrm{O}_{3}{ }^{+}[\mathrm{M}+\mathrm{H}]^{+}: 407.1520$, found: 407.1519 .

1-Phenylhexahydro-1H-pyrrolizine-2-spiro-5'-imidazolidine-2',4'-dione-3-spiro-3"-5-chloroindoline2"-one (4g). White solid; m.p. 193-195 ${ }^{\circ} \mathrm{C} ;{ }^{1} \mathrm{H}-\mathrm{NMR}$ (DMSO-d 6 ): $\delta(\mathrm{ppm}) 1.56-1.71\left(\mathrm{~m}, 1 \mathrm{H}, \mathrm{CH}_{2}\right.$ ), 1.76-1.95 (m, 2H, CH$), 1.96-2.06\left(\mathrm{~m}, 1 \mathrm{H}, \mathrm{CH}_{2}\right), 2.76-2.87\left(\mathrm{~m}, 1 \mathrm{H}, \mathrm{CH}_{2}\right), 3.67(\mathrm{~d}, J=10.4 \mathrm{~Hz}, 1 \mathrm{H}$, $\mathrm{CH}), 4.32-4.44(\mathrm{~m}, 1 \mathrm{H}, \mathrm{CH}), 6.82(\mathrm{~d}, J=8.3 \mathrm{~Hz}, 1 \mathrm{H}, \mathrm{ArH}), 7.22-7.34(\mathrm{~m}, 4 \mathrm{H}, \mathrm{ArH}), 7.33-7.41$ (m, 2H, ArH), 7.49 (d, J=1.9 Hz, 1H, ArH), 8.30 (s, 1H, NH), 10.42 (s, 1H, NH), 10.57 (s, 1H, NH); ${ }^{13} \mathrm{C}\{1 \mathrm{H}\}$-NMR (DMSO-d $)$ ): $\delta 27.50,29.58,46.42,56.67,67.53,76.94,79.70,111.29,125.25,126.33$, $127.51,128.09,128.25,129.43,129.73,134.56,141.09,154.89,172.24,176.10$; HRMS: calcd. for $\mathrm{C}_{22} \mathrm{H}_{19} \mathrm{ClN}_{4} \mathrm{O}_{3}^{+}[\mathrm{M}+\mathrm{H}]^{+}:$423.1225, found: 423.1224 . 
1-Phenylhexahydro-1H-pyrrolizine-2-spiro-5'-imidazolidine-2',4'-dione-3-spiro-3"-N-methylindoline$2^{\prime \prime}$-one (4h). White solid; m.p. 199-201 ${ }^{\circ} \mathrm{C} ;{ }^{1} \mathrm{H}-\mathrm{NMR}$ (DMSO-d 6 ): $\delta$ (ppm) 1.58-1.71 (m, $1 \mathrm{H}, \mathrm{CH}_{2}$ ), 1.73-2.09 (m, 3H, $\left.\mathrm{CH}_{2}\right), 2.80-2.93\left(\mathrm{~m}, 1 \mathrm{H}, \mathrm{CH}_{2}\right), 3.10\left(\mathrm{~s}, 3 \mathrm{H}, \mathrm{CH}_{3}\right), 3.74(\mathrm{~d}, J=10.4 \mathrm{~Hz}, 1 \mathrm{H}, \mathrm{CH})$, 4.38-4.50 (m, 1H, CH), $7.00(\mathrm{~d}, J=7.8 \mathrm{~Hz}, 1 \mathrm{H}, \mathrm{ArH}), 7.06-7.16(\mathrm{~m}, 1 \mathrm{H}, \mathrm{ArH}), 7.22-7.43(\mathrm{~m}, 6 \mathrm{H}$, $\operatorname{ArH}), 7.57(\mathrm{~d}, J=7.5 \mathrm{~Hz}, 1 \mathrm{H}, \mathrm{ArH}), 8.18(\mathrm{~s}, 1 \mathrm{H}, \mathrm{NH}), 10.34(\mathrm{~s}, 1 \mathrm{H}, \mathrm{NH}),{ }^{13} \mathrm{C}\{1 \mathrm{H}\}-\mathrm{NMR}\left(\mathrm{DMSO}-\mathrm{d}_{6}\right)$ : $\delta$ 26.29, 27.28, 29.61, 46.53, 57.04, 67.59, 76.57, 79.58, 108.79, 121.74, 123.65, 127.48, 128.03, 128.28, 129.54, 129.74, 134.78, 143.63, 154.92, 172.40, 174.69; HRMS: calcd. for $\mathrm{C}_{23} \mathrm{H}_{22} \mathrm{~N}_{4} \mathrm{O}_{3}{ }^{+}$ $[\mathrm{M}+\mathrm{H}]^{+}:$403.1767, found: 403.1770 .

1-(3-Chloro)phenylhexahydro-1H-pyrrolizine-2-spiro-5'-imidazolidine-2',4'-dione-3-spiro-3"-indoline2"-one (4i). White solid; m.p. $156-128{ }^{\circ} \mathrm{C} ;{ }^{1} \mathrm{H}-\mathrm{NMR}$ (DMSO- $\left.\mathrm{d}_{6}\right): \delta(\mathrm{ppm}) 1.58-1.71\left(\mathrm{~m}, 1 \mathrm{H}, \mathrm{CH}_{2}\right)$, 1.74-7.97 (m, 3H, $\left.\mathrm{CH}_{2}\right), 2.79-2.91\left(\mathrm{~m}, 1 \mathrm{H}, \mathrm{CH}_{2}\right), 3.74(\mathrm{~d}, J=10.3 \mathrm{~Hz}, 1 \mathrm{H}, \mathrm{CH}), 4.31-4.44(\mathrm{~m}, 1 \mathrm{H}$, $\mathrm{CH}), 6.80(\mathrm{~d}, J=7.7 \mathrm{~Hz}, 1 \mathrm{H}, \mathrm{ArH}), 6.95-7.07$ (m, 1H, ArH), 7.15-7.28 (m, 1H, ArH), 7.29-7.40 (brs, 3H, ArH), 7.45 (s, 1H, ArH), 7.52 (d, J=7.5 Hz, 1H, ArH), 8.32 (s, 1H, NH), 10.40 (s, 1H, NH), $10.43(\mathrm{~s}, 1 \mathrm{H}, \mathrm{NH}) ;{ }^{13} \mathrm{C}\{1 \mathrm{H}\}-\mathrm{NMR}\left(\mathrm{DMSO}_{-} \mathrm{d}_{6}\right): \delta 27.29,29.48,46.44,56.19,67.57,76.76,79.47$, $109.85,121.03,124.07,127.50,128.46,129.40,129.49,130.02,132.89,137.46,142.13,154.98$, 172.21, 176.27; HRMS: calcd. for $\mathrm{C}_{22} \mathrm{H}_{19} \mathrm{ClN}_{4} \mathrm{O}_{3}{ }^{+}[\mathrm{M}+\mathrm{H}]^{+}: 423.1224$, found: 423.1224 .

1-(3-Chloro)phenylhexahydro-1H-pyrrolizine-2-spiro-5'-imidazolidine-2',4'-dione-3-spiro-3"-5chloroindoline-2"-one (4j). White solid; m.p. 161-163 ${ }^{\circ} \mathrm{C}$; ${ }^{1} \mathrm{H}-\mathrm{NMR}$ (DMSO- $\mathrm{d}_{6}$ ): $\delta$ (ppm) 1.64-1.75 $\left(\mathrm{m}, 1 \mathrm{H}, \mathrm{CH}_{2}\right), 1.76-1.94\left(\mathrm{~m}, 2 \mathrm{H}, \mathrm{CH}_{2}\right), 1.95-2.06\left(\mathrm{~m}, 1 \mathrm{H}, \mathrm{CH}_{2}\right), 2.71-2.83\left(\mathrm{~m}, 1 \mathrm{H}, \mathrm{CH}_{2}\right), 3.74(\mathrm{~d}$, $J=10.4 \mathrm{~Hz}, 1 \mathrm{H}, \mathrm{CH}), 4.31-4.38(\mathrm{~m}, 1 \mathrm{H}, \mathrm{CH}), 6.82$ (d, $J=8.3 \mathrm{~Hz}, 1 \mathrm{H}, \mathrm{ArH}), 7.28-7.39$ (m, 4H, ArH), 7.46 (s, 1H, ArH), 7.55 (d, $J=1.7 \mathrm{~Hz}, 1 \mathrm{H}, \mathrm{ArH}), 8.50$ (s, 1H, NH), 10.47 (s, 1H, NH), 10.57 (s, 1H, $\mathrm{NH}) ;{ }^{13} \mathrm{C}\{1 \mathrm{H}\}-\mathrm{NMR}\left(\mathrm{DMSO}_{\mathrm{d}}\right.$ ): $\delta 27.73,29.87,46.88,56.56,68.02,77.02,80.05,109.77,122.27$, $123.78,127.26,128.77,129.08,129.88,130.52$, 133.36, 136.29, 137.46, 142.70, 155.46, 172.43, 175.26; HRMS: calcd. for $\mathrm{C}_{22} \mathrm{H}_{18} \mathrm{Cl}_{2} \mathrm{~N}_{4} \mathrm{O}_{3}{ }^{+}[\mathrm{M}+\mathrm{H}]^{+}$: 457.0831, found: 457.0834 .

1-(3-Chloro)phenylhexahydro-1H-pyrrolizine-2-spiro-5'-imidazolidine-2',4'-dione-3-spiro-3"- $N$ benzylindoline-2"-one (4k). White solid; m.p. 216-218 ${ }^{\circ} \mathrm{C} ;{ }^{1} \mathrm{H}-\mathrm{NMR}$ (DMSO-d $\left.{ }_{6}\right): \delta(\mathrm{ppm}) 1.62-1.75$ $\left(\mathrm{m}, 1 \mathrm{H}, \mathrm{CH}_{2}\right), 1.76-2.04\left(\mathrm{~m}, 3 \mathrm{H}, \mathrm{CH}_{2}\right), 2.79-2.91\left(\mathrm{~m}, 1 \mathrm{H}, \mathrm{CH}_{2}\right), 3.82(\mathrm{~d}, J=10.4 \mathrm{~Hz}, 1 \mathrm{H}, \mathrm{CH})$, 4.40-4.50 (m, 1H, CH), $4.72\left(\mathrm{~d}, J=15.9 \mathrm{~Hz}, 1 \mathrm{H}, \mathrm{CH}_{2}\right), 5.05$ (d, $\left.J=15.9 \mathrm{~Hz}, 1 \mathrm{H}, \mathrm{CH}_{2}\right), 6.83(\mathrm{~d}$, $J=7.8 \mathrm{~Hz}, 1 \mathrm{H}, \operatorname{ArH}), 7.04-7.13(\mathrm{~m}, 1 \mathrm{H}, \operatorname{ArH}), 7.12-7.42(\mathrm{~m}, 9 \mathrm{H}, \operatorname{ArH}), 7.50(\mathrm{~s}, 1 \mathrm{H}, \operatorname{ArH}), 7.62(\mathrm{~d}$, $J=7.5 \mathrm{~Hz}, 1 \mathrm{H}, \mathrm{ArH}), 8.55(\mathrm{~s}, 1 \mathrm{H}, \mathrm{NH}), 10.46(\mathrm{~s}, 1 \mathrm{H}, \mathrm{NH}) ;{ }^{13} \mathrm{C}\{1 \mathrm{H}\}-\mathrm{NMR}\left(\mathrm{DMSO}-\mathrm{d}_{6}\right): \delta 27.68,29.84$, $43.13,46.86,56.63,68.00,77.00,80.02,109.74,122.25,123.76,127.23,127.78,128.03,128.67$, $128.82,129.06,129.78,129.92,130.49,133.35,136.27,137.45,142.69,155.43,172.43,175.24$; HRMS: calcd. for $\mathrm{C}_{29} \mathrm{H}_{25} \mathrm{ClN}_{4} \mathrm{O}_{3}{ }^{+}[\mathrm{M}+\mathrm{H}]^{+}:$513.1693, found: 513.1693.

1-(4-Trifluoromethy)phenylhexahydro-1 H-pyrrolizine-2-spiro-5'-imidazolidine-2',4'-dione-3-spiro-3"indoline-2"-one (4l). White solid; m.p. 239-241 ${ }^{\circ} \mathrm{C}$; ${ }^{1} \mathrm{H}-\mathrm{NMR}\left(\mathrm{DMSO}-\mathrm{d}_{6}\right)$ : $\delta 1.60-1.72\left(\mathrm{~m}, 1 \mathrm{H}, \mathrm{CH}_{2}\right)$, 1.73-1.87 (m, 1H, $\left.\mathrm{CH}_{2}\right), 1.87-2.06\left(\mathrm{~m}, 2 \mathrm{H}, \mathrm{CH}_{2}\right), 2.87-2.98\left(\mathrm{~m}, 1 \mathrm{H}, \mathrm{CH}_{2}\right), 3.82(\mathrm{~d}, J=10.3 \mathrm{~Hz}, 1 \mathrm{H}$, $\mathrm{CH}), 4.41-4.51(\mathrm{~m}, 1 \mathrm{H}, \mathrm{CH}), 6.81(\mathrm{~d}, J=7.7 \mathrm{~Hz}, 1 \mathrm{H}, \mathrm{ArH}), 6.98-7.06(\mathrm{~m}, 1 \mathrm{H}, \mathrm{ArH}), 7.21-7.30$ (m, $1 \mathrm{H}, \operatorname{ArH}), 7.52$ (d, $J=7.6 \mathrm{~Hz}, 1 \mathrm{H}, \mathrm{ArH}), 7.60$ (d, $J=8.2 \mathrm{~Hz}, 2 \mathrm{H}, \operatorname{ArH}), 7.69$ (d, $J=8.2 \mathrm{~Hz}, 2 \mathrm{H}, \operatorname{ArH})$, $8.30(\mathrm{~s}, 1 \mathrm{H}, \mathrm{NH}), 10.45(\mathrm{~s}, 1 \mathrm{H}, \mathrm{NH}), 10.48(\mathrm{~s}, 1 \mathrm{H}, \mathrm{NH}) ;{ }^{13} \mathrm{C}\{1 \mathrm{H}\}-\mathrm{NMR}\left(\mathrm{DMSO}_{-} \mathrm{d}_{6}\right): \delta 27.02,29.38$, 
$46.55,56.55,67.65,76.82,79.50,109.87,121.08,122.92,124.05,125.06,125.62,127.86,128.18$, $128.35,129.50,129.81,130.59,139.88,142.27,154.97,172.52,176.28$; HRMS: calcd. for $\mathrm{C}_{23} \mathrm{H}_{19} \mathrm{~F}_{3} \mathrm{~N}_{4} \mathrm{O}_{3}{ }^{+}[\mathrm{M}+\mathrm{H}]^{+}: 457.1486$, found: 457.1488 .

1-(3-Methoxy)phenylhexahydro-1H-pyrrolizine-2-spiro-5'-imidazolidine-2',4'-dione-3-spiro-3"-indoline2"-one (4m). Yellow solid; m.p. $177-179{ }^{\circ} \mathrm{C} ;{ }^{1} \mathrm{H}-\mathrm{NMR}$ (400 MHz, DMSO-d6): $\delta$ (ppm) 1.57-1.70 (m, 1H, $\left.\mathrm{CH}_{2}\right), 1.72-1.87\left(\mathrm{~m}, 1 \mathrm{H}, \mathrm{CH}_{2}\right), 1.87-2.04\left(\mathrm{~m}, 2 \mathrm{H}, \mathrm{CH}_{2}\right), 2.83-2.95\left(\mathrm{~m}, 1 \mathrm{H}, \mathrm{CH}_{2}\right), 3.67$ (d, $J=10.3 \mathrm{~Hz}, 1 \mathrm{H}, \mathrm{CH}), 4.35-4.46(\mathrm{~m}, 1 \mathrm{H}, \mathrm{CH}), 6,77-6.86(\mathrm{~m}, 2 \mathrm{H}, \mathrm{ArH}), 6.89$ (d, J=7.6 Hz, $1 \mathrm{H}, \mathrm{ArH})$, 6.97-7.06 (m, 2H, ArH), 7.16-7.28 (m, 2H, ArH), 7.49 (d, J=7.5 Hz, 1H, ArH), 8.07 (s, 1H, NH), $10.34(\mathrm{~s}, 1 \mathrm{H}, \mathrm{NH}), 10.40(\mathrm{~s}, 1 \mathrm{H}, \mathrm{NH}) ;{ }^{13} \mathrm{C}\{1 \mathrm{H}\}-\mathrm{NMR}(100 \mathrm{MHz}, \mathrm{DMSO}-\mathrm{d} 6): \delta 27.16,29.63,46.50$, 54.93, 56.85, 59.75, 67.50, 76.83, 79.45, 109.82, 112.82, 115.22, 121.01, 121.95, 124.25, 128.34, $129.25,136.42,142.21,155.00,159.07,172.55,176.43$; HRMS: calcd. for $\mathrm{C}_{23} \mathrm{H}_{22} \mathrm{~N}_{4} \mathrm{O}_{4}{ }^{+}[\mathrm{M}+\mathrm{H}]+$ : 419.1718, found: 419.1719 .

7-Phenyl-5-spiro-3'-indoline-2'-one-hexahydropyrrolo[1,2-c] thiazole-6-spiro-5"-imidazolidine-2", 4"dione (4n). White solid; m.p. 200-202 ${ }^{\circ} \mathrm{C}$; ${ }^{1} \mathrm{H}-\mathrm{NMR}$ (DMSO-d6): $\delta$ (ppm) 2.87 (dd, $J=11.2,4.1 \mathrm{~Hz}$, $\left.1 \mathrm{H}, \mathrm{CH}_{2}\right) .3 .07$ (dd, $\left.J=11.1,6.8 \mathrm{~Hz}, 1 \mathrm{H}, \mathrm{CH}_{2}\right), 3.54$ (d, $\left.J=9.4 \mathrm{~Hz}, 1 \mathrm{H}, \mathrm{CH}\right), 3.71-3.80\left(\mathrm{~m}, 2 \mathrm{H}, \mathrm{CH}_{2}\right)$, $4.63(\mathrm{~m}, 1 \mathrm{H}, \mathrm{CH}), 6.79$ (d, J=7.7 Hz, 1H, ArH), 7.01 (m, 1H, ArH), 7.21-7.35 (m, 4H, ArH), 7.40 (d, $J=6.6 \mathrm{~Hz}, 1 \mathrm{H}, \mathrm{ArH}), 7.73(\mathrm{~d}, J=7.6 \mathrm{~Hz}, 1 \mathrm{H}, \mathrm{ArH}), 8.58(\mathrm{~s}, 1 \mathrm{H}, \mathrm{NH}), 10.30(\mathrm{~s}, 1 \mathrm{H}, \mathrm{NH}), 10.48$ (s, $1 \mathrm{H}, \mathrm{NH}) ;{ }^{13} \mathrm{C}\{1 \mathrm{H}\}-\mathrm{NMR}\left(\mathrm{DMSO}_{-} \mathrm{d}_{6}\right): \delta 34.80,51.90,55.13,70.18,77.11,78.29,109.70,120.70$, 122.78, 127.84, 128.31, 129.21, 129.67, 129.83, 133.78, 141.79, 154.84, 170.67, 175.45; HRMS: calcd. for $\mathrm{C}_{21} \mathrm{H}_{18} \mathrm{~N}_{4} \mathrm{O}_{3} \mathrm{~S}^{+}[\mathrm{M}+\mathrm{H}]^{+}$: 407.1176 , found: 407.1178 .

\subsection{Crystallographic Data and Molecular Structure of Compound 4b [32]}

$\mathrm{C}_{29} \mathrm{H}_{26} \mathrm{~N}_{4} \mathrm{O}_{3} \cdot \mathrm{C}_{2} \mathrm{H}_{6} \mathrm{O}, \mathrm{M}=524.61$, triclinic, $\mathrm{a}=9.8656(7) \AA, \mathrm{b}=11.0707(8) \AA, \mathrm{c}=13.5643(10) \AA, \alpha$ $=98.6770(10)^{\circ}, \beta=110.6190(10)^{\circ}, \gamma=103.4270(10)^{\circ}, \mathrm{V}=1303.90(16) \AA 3, \mathrm{~T}=100(2) \mathrm{K}$, space group $\mathrm{P} \overline{1}, \mathrm{Z}=2, \mu(\mathrm{MoK} \alpha)=0.090 \mathrm{~mm}-1,18612$ reflections measured, 7266 independent reflections $($ Rint $=0.0225)$. The final R1 values were $0.0436(I>2 \sigma(I))$. The final $w R(F 2)$ values were 0.1160 $(\mathrm{I}>2 \sigma(\mathrm{I})$ ). The final R1 values were 0.0560 (all data). The final wR(F2) values were 0.1250 (all data). The goodness of fit on F2 was 1.021 .

\section{Conclusions}

In conclusion, we have successfully developed an efficient method for the synthesis of potentially biologically active a series of novel dispirocycloadducts via a three-component 1,3-dipolar cycloaddition reaction of azomethine ylides. This method has the advantages of convenient operation, the availability of starting materials, mild reaction conditions employed, high yields and high efficiency, as well as the complete regio- and stereoselectivity observed. Further studies to acquire more information about the pharmacological activity of these compounds are in progress in our laboratory.

\section{Supplementary Materials}

Supplementary materials can be accessed at: http://www.mdpi.com/1420-3049/18/5/5142/s1. 


\section{Acknowledgments}

We are grateful to Zhiyan Huang (Nanyang Technological University) for his critical reviews on this manuscript. We also thank Xiaonian Li (Kunming Institute of Botany) and Jian Yang (Kunming University of Science and Technology) for their helpful assistance with the single crystal X-ray diffraction analysis. Financial support from Natural Science Foundation of China (No. 81102325), China Postdoctoral Science Foundation (No. 2012T50781), Young Teachers Fund of Sichuan University (No. 2011SCU11108) and Sichuan Provincial Health Department Research Project (No. 110173) is gratefully acknowledged.

\section{Conflict of Interest}

The authors declare no conflict of interest.

\section{References and Notes}

1. Pellissier, H. Stereocontrolled domino reactions. Chem. Rev. 2013, 113, 442-524.

2. Slobbe, P.; Ruijter, E.; Orru, R.V.A. Recent applications of multicomponent reactions in medicinal chemistry. Medchemcomm 2012, 3, 1189-1218.

3. Domling, A.; Wang, W.; Wang, K., Chemistry and biology of multicomponent reactions. Chem. Rev. 2012, 112, 3083-3135.

4. Bonne, D.; Constantieux, T.; Coquerel, Y.; Rodriguez, J. Stereoselective Multiple Bond-Forming Transformations (MBFTs): The Power of 1,2-and 1,3-Dicarbonyl Compounds. Chem. Eur. J. 2013, 19, 2218-2231.

5. Burrell, A.J.M.; Coldham, I.; Watson, L.; Oram, N.; Pilgram, C.D.; Martin, N.G. Stereoselective formation of fused tricyclic amines from acyclic aldehydes by a cascade process involving condensation, cyclization, and dipolar cycloaddition. J. Org. Chem. 2009, 74, 2290-2300.

6. Singh, G.S.; Desta, Z.Y. Isatins as privileged molecules in design and synthesis of spiro-fused cyclic frameworks. Chem. Rev. 2012, 112, 6104-6155.

7. Nair, V.; Suja, T.D. Intramolecular 1,3-dipolar cycloaddition reactions in targeted syntheses. Tetrahedron 2007, 63, 12247-12275.

8. Presset, M.; Mohanan, K.; Hamann, M.; Coquerel, Y.; Rodriguez, J. 1,3-Dipolar Cycloaddition of Hydrazones with alpha-Oxo-ketenes: A Three-Component Stereoselective Entry to Pyrazolidinones and an Original Class of Spirooxindoles. Org. Lett. 2011, 13, 4124-4127.

9. Lashgari, N.; Ziarani, G.M. Synthesis of heterocyclic compounds based on isatin through 1, 3-dipolar cycloaddition reactions. ARKIVOC 2012, 277-320.

10. Liu, H.; Dou, G.L.; Shi, D.Q. Regioselective synthesis of novel spiropyrrolidines and spirothiapyrrolizidines through multicomponent 1,3-Dipolar cycloaddition reaction of azomethine ylides. J. Comb. Chem. 2010, 12, 633-637.

11. Liu, H.; Dou, G.L.; Shi, D.Q. Regio- and stereoselective synthesis of novel dispiropyrrolidine bisoxindole derivatives via multicomponent reactions. J. Comb. Chem. 2010, 12, 292-294. 
12. Hu, Y.; Zou, Y.; Wu, H.; Shi, D.Q. A facile and efficient ultrasound-assisted synthesis of novel dispiroheterocycles through 1,3-dipolar cycloaddition reactions. Ultrason. Sonochem. 2012, 19, 264-269.

13. Huang, Z.B.; Zhao, Q.; Chen, G.; Wang, H.Y.; Lin, W.; Xu, L.X.; Liu, H.T.; Wang, J.X.; Shi, D.Q.; Wang, Y.C. An efficient synthesis of novel dispirooxindole derivatives via one-pot three-component 1,3-Dipolar cycloaddition reactions. Molecules 2012, 17, 12704-12717.

14. Arun, Y.; Bhaskar, G.; Balachandran, C.; Ignacimuthu, S.; Perumal, P.T. Facile one-pot synthesis of novel dispirooxindole-pyrrolidine derivatives and their antimicrobial and anticancer activity against A549 human lung adenocarcinoma cancer cell line. Bioorgan. Med. Chem. Lett. 2013, 23, 1839-1845.

15. Tian, Y.; Nam, S.; Liu, L.; Yakushijin, F.; Yakushijin, K.; Buettner, R.; Liang, W.; Yang, F.; Ma, Y.L.; Horne, D.; Jove, R. Spirooxindole derivative SOID-8 induces apoptosis associated with inhibition of JAK2/STAT3 signaling in melanoma cells. PLOS One 2012, 7, e49306.

16. Chowdhury, S.; Liu, S.F.; Cadieux, J.A.; Hsieh, T.; Chafeev, M.; Sun, S.Y.; Jia, Q.; Sun, J.Y.; Wood, M.; Langille, J.; et al. Tetracyclic spirooxindole blockers of $\mathrm{hNa}(\mathrm{V}) 1.7$ : Activity in vitro and in CFA-induced inflammatory pain model. Med. Chem. Res. 2013, 22, 1825-1836.

17. Nicolaou, K.C.; Sanchini, S.; Sarlah, D.; Lu, G.; Wu, T.R.; Nomura, D.K.; Cravatt, B.F.; Cubitt, B.; de la Torre, J.C.; Hessell, A.J. et al. Design, synthesis, and biological evaluation of a biyouyanagin compound library. Proc. Natl. Acad. Sci. USA 2011, 108, 6715-6720.

18. Millard, M.; Pathania, D.; Grande, F.; Xu, S.L.; Neamati, N. Small-Molecule Inhibitors of p53MDM2 Interaction: The 2006-2010 Update. Curr. Pharm. Des. 2011, 17, 536-559.

19. Palnitkar, S.S.; Bin, B.; Jimenez, L.S.; Morimoto, H.; Williams, P.G.; Paul-Pletzer, K.; Parness, J. H-3 Azidodantrolene: Synthesis and use in identification of a putative skeletal muscle dantrolene binding site in sarcoplasmic reticulum. J. Med. Chem. 1999, 42, 1872-1880.

20. Byrtus, H.; Obniska, J.; Czopek, A.; Kaminski, K.; Pawlowski, M. Synthesis and anticonvulsant activity of new N-Mannich bases derived from 5-cyclopropyl-5-phenyl- and 5-cyclopropyl-5-(4chlorophenyl)-imidazolidine-2,4-diones. Bioorg. Med. Chem. 2011, 19, 6149-6156.

21. Blanco-Ania, D.; Hermkens, P.H.H.; Sliedregt, L.; Scheeren, H.W.; Rutjes, F. Synthesis of hydantoins and thiohydantoins spiro-fused to pyrrolidines: Druglike molecules based on the 2-Arylethyl amine scaffold. J. Comb. Chem. 2009, 11, 527-538.

22. Basappa; Kumar, C.S.A.; Swamy, S.N.; Sugahara, K.; Rangappa, K.S. Anti-tumor and anti-angiogenic activity of novel hydantoin derivatives: Inhibition of VEGF secretion in liver metastatic osteosarcoma cells. Bioorg. Med. Chem. 2009, 17, 4928-4934.

23. Khanfar, M.A.; Hill, R.A.; Kaddoumi, A.; El Sayed, K.A. Discovery of novel GSK-3 beta inhibitors with potent in vitro and in vivo activities and excellent brain permeability using combined ligand- and structure-based virtual screening. J. Med. Chem. 2010, 53, 8534-8545.

24. Zhang, D.Y.; Ye, D.J.; Feng, E.G.; Wang, J.F.; Shi, J.M.; Jiang, H.L.; Liu, H. Highly alphaselective synthesis of sialyl spirohydantoins by regiospecific domino condensation/O $->\mathrm{N}$ acyl migration/ $\mathrm{N}$-sialylation of carbodiimides with peracetylated sialic acid. J. Org. Chem. 2010, 75, 3552-3557. 
25. Merino-Montiel, P.; Lopez, O.; Alvarez, E.; Fernandez-Bolanos, J.G. Synthesis of conformationally-constrained thio(seleno)hydantoins and alpha-triazolyl lactones from D-arabinose as potential glycosidase inhibitors. Tetrahedron 2012, 68, 4888-4898.

26. Xie, Y.M.; Yao, Y.Q.; Sun, H.B.; Yan, T.T.; Liu, J.; Kang, T.R. Facile synthesis of functionalized spiropyrrolizidine oxindoles via a three-component tandem cycloaddition reaction. Molecules 2011, 16, 8745-8757.

27. Liu, J.; Sun, H.B.; Liu, X.J.; Ouyang, L.; Kang, T.R.; Xie, Y.M.; Wang, X.Y. Direct construction of novel exo'-selective spiropyrrolidine bisoxindoles via a three-component 1,3-dipolar cycloaddition reaction. Tetrahedron Lett. 2012, 53, 2336-2340.

28. Wu, G.S.; Ouyang, L.; Liu, J.; Zeng, S.; Huang, W.; Han, B.; Wu, F.B.; He, G.; Xiang, M.L. Synthesis of novel spirooxindolo-pyrrolidines, pyrrolizidines, and pyrrolothiazoles via a regioselective three-component [3+2] cycloaddition and their preliminary antimicrobial evaluation. Mol. Divers. 2013, 17, 271-283.

29. Thenmozhiyal, J.C.; Wong, P.T.H.; Chui, W.K. Anticonvulsant activity of phenylmethylenehydantoins: A structure-activity relationship study. J. Med. Chem. 2004, 47, 1527-1535.

30. Pardasani, R.T.; Pardasani, P.; Chaturvedi, V.; Yadav, S.K.; Saxena, A.; Sharma, I. Theoretical and synthetic approach to novel spiroheterocycles derived from isatin derivatives and L-proline via 1,3-dipolar cycloaddition. Heteroatom Chem. 2003, 14, 36-41.

31. Lakshmi, N.V.; Thirumurugan, P.; Perumal, P.T. An expedient approach for the synthesis of dispiropyrrolidine bisoxindoles, spiropyrrolidine oxindoles and spiroindane-1,3-diones through 1,3-dipolar cycloaddition reactions. Tetrahedron Lett. 2010, 51, 1064-1068.

32. Crystallographic data of compooud $\mathbf{4 b}$ reported in this manuscript have been deposited with Cambridge Crystallographic Data Centre as supplement ary publication no. CCDC-927973. Available online: http://www.ccdc. cam.ac.uk/conts/retrieving.html (accessed on 3 May 2013).

Sample Availability: Samples of all the compounds are available from the authors.

(C) 2013 by the authors; licensee MDPI, Basel, Switzerland. This article is an open access article distributed under the terms and conditions of the Creative Commons Attribution license (http://creativecommons.org/licenses/by/3.0/). 